\title{
Storage of Colour Information in a Greyscale Image using Haar Wavelets and Various Colour Spaces
}

\author{
Dr. H. B. Kekre \\ Senior Professor, \\ Computer Engineering \\ Department, \\ Mukesh Patel School of \\ Technology Management and \\ Engineering, \\ SVKM's NMIMS University, \\ Mumbai, India
}

\author{
Sudeep D. Thepade \\ Ph. D. Research Scholar and \\ Associate Professor, \\ Computer Engineering \\ Department, \\ Mukesh Patel School of \\ Technology Management and \\ Engineering, \\ SVKM's NMIMS University, \\ Mumbai, India
}

\author{
Adib Parkar \\ B. E. Computer Student \\ Thadomal Shahani Engineering \\ College, \\ Mumbai University, \\ Mumbai, India
}

\begin{abstract}
The technique of embedding colour information in a greyscale image such that the original colour image can be reconstructed from this modified greyscale image has previously been proposed using the $\mathrm{YCbCr}$ colour space with the improvement using Kekre's LUV colour space. The embedding of the colour information into the greyscale image leads to the formation of a slightly distorted greyscale image known as a "matted greyscale" image. This matted greyscale image is used to reconstruct the colour image. Such a technique can be used effectively for image compression as the matted greyscale image alone can be used to reconstruct the original colour image. Also the technique enables transmission of colour documents over black and white fax machine. This paper extends this technique to other colour spaces like Kekre's $\mathrm{YCgCb}$, YIQ and YUV, and employs the Haar transform. For the performance analysis of the technique is applied on 20 different images. The mean squared error (MSE) difference between original grey and matted gray along with MSE difference in original colour and recoloured image play role of statistical performance measures. From the results the conclusions drawn are Kekre's LUV colour space gives best recolouring and Kekre's $\mathrm{YCgCb}$ colour space gives minimum distortion in matted grey image.
\end{abstract}

Keywords - Colouring, Colour to Grey, Matted Greyscale, YCbCr, Kekre's LUV, Kekre's YCgCb, YIQ, YUV, Colour Spaces, Haar Wavelets.

\section{INTRODUCTION}

Two of the greatest conundrums in digital image processing today are how to minimize the storage requirements for coloured images, and how to retrieve a colour image from a greyscale image, that is, how to colour a greyscale image. Minimization of storage requirements for coloured images has been studied extensively and many compression algorithms exist, both lossy and lossless, that perform extremely well. In fact, many of these algorithms have been standardized and are actively used today, such as the JPEG algorithm. On the other hand, the colouring of greyscale images poses more problems, and this is still an area of active research. Many techniques have been proposed, such as colouring using seed pixels, colouring using statistical analysis [1], and even automatic colouring using colour maps [2].

This paper describes a technique to embed colour information in a greyscale image thus providing a straightforward procedure to reconstruct the colour image from the greyscale image and indirectly also provide image compression. The compression is achieved as the colour information is now stored in a greyscale image which inherently requires less storage space than its colour equivalent. Such a greyscale image that contains embedded colour information is referred to as a "matted greyscale" image.
Hiding the colour information inside the greyscale image can lead to even more interesting applications. Greyscale images can be printed out using printers that use only black ink. Hence, a matted greyscale image can also be printed using such a printer. If one wishes to reconstruct the colour image from this matted greyscale printout, one simply has to scan the image, that is recreate the digital equivalent of the matted greyscale image, and extract the colour information from it. Then, if a colour printer is available, a coloured version of the image can also be printed out if desired.

The process of embedding the colour information into a greyscale image and its subsequent retrieval to recreate a coloured image is based on a technique proposed in [3] and [7]. The general procedure is as follows. First, the original colour image is converted to its greyscale equivalent in some colour space. This image is then transformed using an appropriate image transform into the transform domain. In the transform domain, the transformed image is modified by embedding the colour components of the image. These components are embedded by replacing the lesser significant portions (high frequency regions) of the transformed image by scaled down versions of the colour components. The most significant portion (low frequency region) of the transformed image is, of course, left untouched. This modified transformed image is then inverse transformed back into the spatial domain. The greyscale image so obtained is known as the matted greyscale image. Reconstructing the colour image from this matted greyscale image is now straightforward. One simply has to apply the transform, extract the scaled down versions of the colour components, scale them up to their original size, and create a colour image using these scaled up components. Of course, a certain amount of information will be lost due to this scaling of the components.

While the procedure used in [3] involved using the Haar Wavelet Transform [12] along with YCbCr colour space and improvement given in [7] used Kekre's LUV colour space, here along with those two some more colour spces are used like YUV, YIQ and Kekre's $\mathrm{YCgCb}$.

The following sections describe the colour spaces used, followed by a detailed description of the steps involved in this technique, and ending with a comparison of the results obtained using the five colour spaces. Thus an exhaustive comparison across the various colour spaces is obtained in this paper.

\section{II. $\mathrm{YCbCr}$ COLOUR SPACE}

The YCbCr model defines a colour space in terms of one luminance (brightness) and two chrominance (colour) components. It is one of the most extensively used colour spaces and has been considered for many applications such as those described in [3], [4], and [5]. In the $\mathrm{YCbCr}$ colour space, the $\mathrm{Y}$ component gives luminance and the $\mathrm{Cb}$ and $\mathrm{Cr}$ components give the chromaticity 
values of the colour image. To get the $\mathrm{YCbCr}$ components, the conversion of the RGB components to $\mathrm{YCbCr}$ components must be known. The RGB to YCbCr conversion matrix is given below.

$$
\left[\begin{array}{c}
Y \\
C b \\
C r
\end{array}\right]=\left[\begin{array}{ccc}
0.2989 & 0.5866 & 0.1145 \\
-0.1688 & -0.3312 & 0.5 \\
0.5 & -0.4184 & -0.0816
\end{array}\right] \cdot\left[\begin{array}{l}
R \\
G \\
B
\end{array}\right]
$$

To get the RGB values from the $\mathrm{YCbCr}$ components, the following conversion matrix can be used.

$$
\left[\begin{array}{l}
R \\
G \\
B
\end{array}\right]=\left[\begin{array}{ccc}
1 & -0.001 & 1.402 \\
1 & -0.3441 & -0.714 \\
1 & 1.7718 & 0.001
\end{array}\right] \cdot\left[\begin{array}{c}
Y \\
C b \\
C r
\end{array}\right]
$$

\section{Kekre's LUV COLOUR SPACE}

The Kekre's LUV colour space is a colour space generally used in techniques involving the colourization of images such as those described in [2], [6]-[8] and [14]. In the Kekre's LUV colour space, the L component provides the luminance, while the $\mathrm{U}$ and $\mathrm{V}$ components contain the colour information. The RGB to Kekre's LUV conversion matrix is given below.

$$
\left[\begin{array}{l}
L \\
U \\
V
\end{array}\right]=\left[\begin{array}{ccc}
1 & 1 & 1 \\
-2 & 1 & 1 \\
0 & -1 & 1
\end{array}\right] \cdot\left[\begin{array}{l}
R \\
G \\
B
\end{array}\right]
$$

To get the RGB values from the Kekre's LUV components, the following conversion matrix can be used.

$$
\left[\begin{array}{l}
R \\
G \\
B
\end{array}\right]=\left[\begin{array}{ccc}
1 & -2 & 0 \\
1 & 1 & -1 \\
1 & 1 & 1
\end{array}\right] \cdot\left[\begin{array}{l}
L / 3 \\
U / 6 \\
V / 2
\end{array}\right]
$$

A negative value for the U component in the Kekre's LUV colour space indicates prominence of the red component in the colour image. Similarly, a negative value for the V component indicates prominence of the green component over the blue component in the colour image.

\section{YCgCb COLOUR SPACE}

The Kekre's YCgCb colour model is a newly proposed colour space similar to the Kekre's LUV colour space described in the previous section. Since it is newer than the Kekre's LUV colour space, it has not yet been used extensively.

In the Kekre's $\mathrm{YCgCb}$ colour space, the $\mathrm{Y}$ component provides the luminance, while the $\mathrm{Cg}$ and $\mathrm{Cb}$ components contain the chromaticity values. The RGB to Kekre's $\mathrm{YCgCb}$ conversion matrix is given below.

$$
\left[\begin{array}{c}
Y \\
C g \\
C b
\end{array}\right]=\left[\begin{array}{ccc}
1 & 1 & 1 \\
1 & -1 & 0 \\
1 & 0 & -1
\end{array}\right] \cdot\left[\begin{array}{l}
R \\
G \\
B
\end{array}\right]
$$

To get the RGB values from the Kekre's YCgCb components, the following conversion matrix can be used.

$$
\left[\begin{array}{l}
R \\
G \\
B
\end{array}\right]=\left[\begin{array}{ccc}
1 & 1 & 1 \\
1 & -1 & 0 \\
1 & 0 & -1
\end{array}\right] \cdot\left[\begin{array}{c}
Y / 3 \\
C g / 2 \\
C b / 2
\end{array}\right]
$$

A negative value for the $\mathrm{Cg}$ component in the Kekre's $\mathrm{YCgCb}$ colour space indicates prominence of the green component over the red component in the colour image. Similarly, a negative value for the $\mathrm{Cb}$ component indicates prominence of the blue component over the red component.

\section{YIQ COLOUR SPACE}

YIQ is the colour space used by the NTSC colour TV system [15], employed mainly in North and Central America. 'I' stands for "in phase" and 'Q' stands for "quadrature," referring to the components used in quadrature amplitude modulation.

As in the $\mathrm{YCbCr}$ colour space, the $\mathrm{Y}$ component gives luminance and the I and Q components give the chromaticity values of the colour image. To get the YIQ components, the conversion of the RGB components to the YIQ components is defined by the following conversion matrix.

$$
\left[\begin{array}{l}
Y \\
I \\
Q
\end{array}\right]=\left[\begin{array}{ccc}
0.299 & 0.587 & 0.114 \\
0.596 & -0.275 & -0.321 \\
0.212 & -0.523 & 0.311
\end{array}\right] \cdot\left[\begin{array}{l}
R \\
G \\
B
\end{array}\right]
$$

To get the RGB values from the YIQ components, the following conversion matrix can be used.

$$
\left[\begin{array}{l}
R \\
G \\
B
\end{array}\right]=\left[\begin{array}{ccc}
1 & 0.956 & 0.621 \\
1 & -0.272 & -0.647 \\
1 & -1.107 & 1.704
\end{array}\right] \cdot\left[\begin{array}{l}
Y \\
I \\
Q
\end{array}\right]
$$

\section{YUV COLOUR SPACE}

YUV is a colour space that encodes a colour image or video taking human perception into account, allowing reduced bandwidth for chrominance, thereby typically enabling transmission errors or compression artefacts to be more efficiently masked by human perception than using a direct RGB representation.

Similar to the other colour spaces discussed previously, the $\mathrm{Y}$ component gives luminance, and the $\mathrm{U}$ and $\mathrm{V}$ components provide the chrominance values. The RGB to YUV conversion matrix is shown below.

$$
\left[\begin{array}{l}
Y \\
U \\
V
\end{array}\right]=\left[\begin{array}{ccc}
0.299 & 0.587 & 0.114 \\
-0.1471 & -0.2889 & 0.436 \\
0.615 & -0.5149 & 0.10001
\end{array}\right] \cdot\left[\begin{array}{l}
R \\
G \\
B
\end{array}\right]
$$

To get the RGB values from the YUV components, the following conversion matrix can be used.

$$
\left[\begin{array}{l}
R \\
G \\
B
\end{array}\right]=\left[\begin{array}{ccc}
0.74952 & -0.509 & 1.1398 \\
1.0836 & -0.2247 & -0.5806 \\
0.97086 & 1.9729 & 0.00001467
\end{array}\right] \cdot\left[\begin{array}{c}
Y \\
U \\
V
\end{array}\right]
$$

\section{COLOUR TO MATTED GREY CONVERSION}

The most trivial way to convert a colour image to greyscale for printing is to retain and use the luminance component of the colour image. The problem with this approach is that regions that have contrasting colours with similar luminance components would be assigned the same output luminance level and would, therefore, look the same.

The other option is to map colours to textures [3]. One can control halftone dots or patterns as a function of the colours, for example, as a function of hue and saturation. Hence, regions of different colours with similar luminance will look different after mapping because they would have different textures [10]. The procedure proposed in [3] and [7] produces a continuum of textures using the DWT that naturally switch between patterns without causing visual artefacts. The DWT decomposes an image into several sub-bands [11] each representing different spatial frequency contents. 
Here various colour spaces are used hence the procedure is outlined for a general colour space ' $\mathrm{ABC}$ ' where ' $\mathrm{A}$ ' is the luminance component, and ' $\mathrm{B}$ ' and ' $\mathrm{C}$ ' are the chromaticity components. The transform used, as mentioned previously, is the Haar transform.

The steps involved to create the matted greyscale image are as follows.

1. The original colour image is converted from the RGB colour space into the $\mathrm{ABC}$ colour space using the appropriate conversion matrix, that is, using equations (1), (3), (5), (7), or (9) for the YCbCr, Kekre's LUV, Kekre's YCgCb, YIQ, and YUV colour spaces respectively. The 'A' component is the luminance and is considered as the "original greyscale" image.

2. The ' $A$ ' component is transformed using the Haar transform [12],[13] into the transform domain. Let this transformed image be known as $\mathrm{T}_{\mathrm{o}}$.

3. $\mathrm{T}_{\mathrm{o}}$ can be divided into 4 regions as shown in figure 1 below.

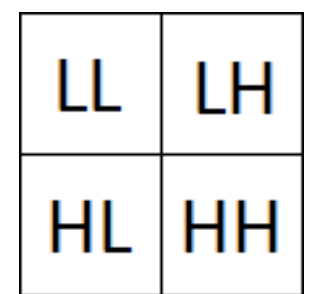

Figure $1-$ Structure of $\mathrm{T}_{\mathrm{o}}$

4. Most of the information in the image is found in the LL region of To which corresponds to the low frequency components of the image. This region is left untouched. The LH and HL regions of To are replaced by scaled down versions of the ' $\mathrm{B}$ ' and ' $\mathrm{C}$ ' components respectively. Thus we now have a modified transformed image that contains scaled down versions of the chromaticity components of the colour space being used. Let this be known as Tm.

5. The inverse Haar transform is now applied to Tm to get a new greyscale image in the spatial domain. This greyscale image now contains colour information hidden within its transform and is known as the matted greyscale image.

VIII. COLOUR EXTRACTION FROM MATTED GREY Since the matted greyscale image already contains the colour information hidden within its transform, extracting the colour image from the matted greyscale image is a straightforward procedure. It consists of the following steps.

1. The matted greyscale image is read or scanned.

2. Once available in digital form, the matted greyscale image is transformed using the Haar transform into the transform domain. The transformed image obtained will be $\mathrm{T}_{\mathrm{m}}$.

3. $\mathrm{T}_{\mathrm{m}}$ can be represented as shown in figure 2 below.

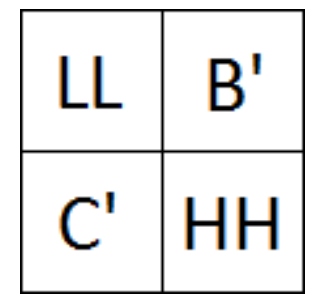

Figure $2-$ Structure of $T_{m}$

4. The $\mathrm{B}$ ' and $\mathrm{C}$ ' regions of $\mathrm{T}_{\mathrm{m}}$ are the scaled down versions of the ' $\mathrm{B}$ ' and ' $\mathrm{C}$ ' components of the original colour image. Thus by extracting these two regions from $\mathrm{T}_{\mathrm{m}}$ and scaling them up back to their original size we get $\mathrm{B}_{\text {approx }}$ and $\mathrm{C}_{\text {approx }}$ which are approximations of the original ' $B$ ' and ' $C$ ' components of the original colour image.

5. To retrieve an approximation for the ' $A$ ' component, we replace regions $\mathrm{B}^{\text {' and }} \mathrm{C}^{\prime}$ in $\mathrm{T}_{\mathrm{m}}$ by zeroes and perform an inverse Haar transformation. The image obtained in the spatial domain is an approximation of the original ' $A$ ' component of the image, $\mathrm{A}_{\text {approx }}$.

6. Now the approximations for the $\mathrm{ABC}$ components are used to convert the image back to the RGB colour space using the appropriate conversion matrices, that is, equations (2), (4), (6), (8), and (10) for the YCbCr, Kekre's LUV, Kekre's YCgCb, YIQ, and YUV colour spaces respectively.

\section{IMPLEMENTATION AND RESULTS}

The technique described in the previous section was applied to 20 images of the size $256 \times 256$ pixels which belonged to various categories such as people, objects, vehicles, animals, cartoons, and nature.

On applying the technique proposed, the following set of images was derived from the each original image - original colour, original greyscale, matted greyscale, and reconstructed colour.

Performance was measured by calculating the mean square error (MSE) between the original greyscale image and the matted greyscale image as well as between the original colour image and the reconstructed colour image. The Mean Squared Error (MSE) between an image I and image $\mathrm{Q}$ can be given as the equation below.

$$
M S E=\frac{1}{n^{2}} \sum_{i=1}^{n} \sum_{j=1}^{n}[I(i, j)-Q(i, j)]^{2}
$$

It must be noted that for a colour image, each pixel will have 3 values, one for the red $(\mathrm{R})$ plane, one for the green $(\mathrm{G})$ plane, and one for the blue (B) plane.

The MSE thus provides an objective criterion that can be used as a measure of the degree of similarity between two given images. The greater the similarity between the two images at the pixel level, the lower the MSE.

The 20 images used for experimentation when implementing this procedure are shown in figure 3.

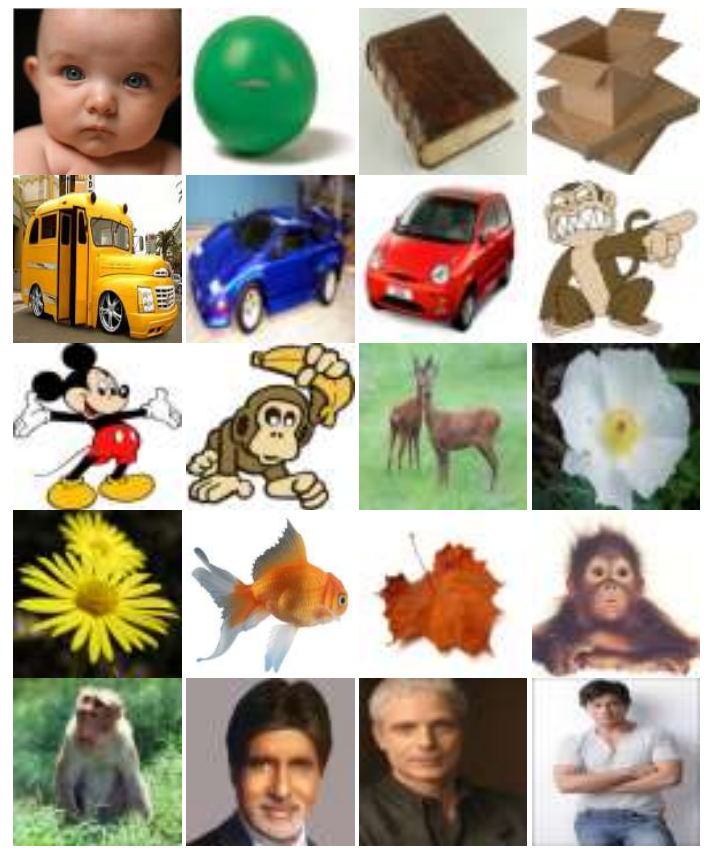

Figure 3 - Images used to implement this procedure 
The table 1 given below shows the MSE values between the matted greyscale images and the original greyscale images averaged across all images for each of the five colour spaces. The figure 4 illustrates the results of table 1 graphically.

\begin{tabular}{|c|c|}
\hline Colour Space & MSE \\
\hline YCbCr & 411.2213 \\
\hline Kekre's LUV & 341.3241 \\
\hline Kekre's YCgCb & 317.0061 \\
\hline YIQ & 432.7004 \\
\hline YUV & 643.3803 \\
\hline
\end{tabular}

Table 1 - Greyscale MSE across all images

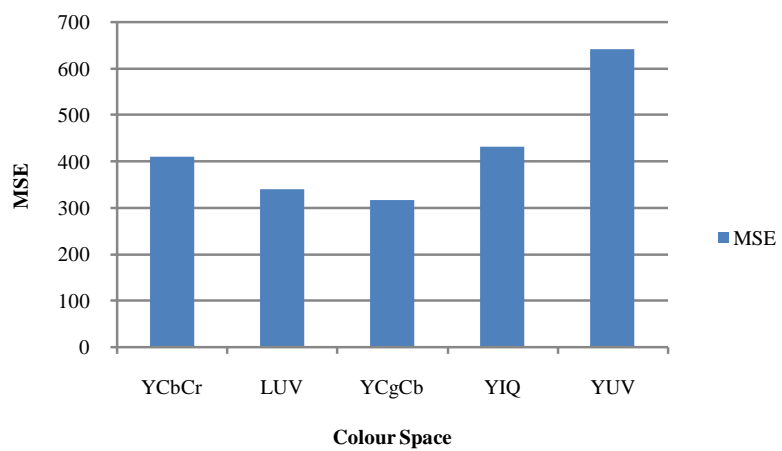

Figure 4-Average MSE between original greyscale and matted greyscale images

As can be clearly seen from the figure 4, the Kekre's $\mathrm{YCgCb}$ colour space provided the best results in terms of the MSE across all images for the matted greyscale image.

The results for the reconstructed colour images, however, were slightly different. The table 2 shows the MSE values between the reconstructed colour images and the original colour images. Figure 5 illustrates the results of table 2 graphically.

\begin{tabular}{|c|c|}
\hline Colour Space & MSE \\
\hline YCbCr & 245.3661 \\
\hline Kekre's LUV & 233.0012 \\
\hline Kekre's YCgCb & 245.3116 \\
\hline YIQ & 249.9037 \\
\hline YUV & 417.9223 \\
\hline
\end{tabular}

Table 2 - Colour MSE across all images

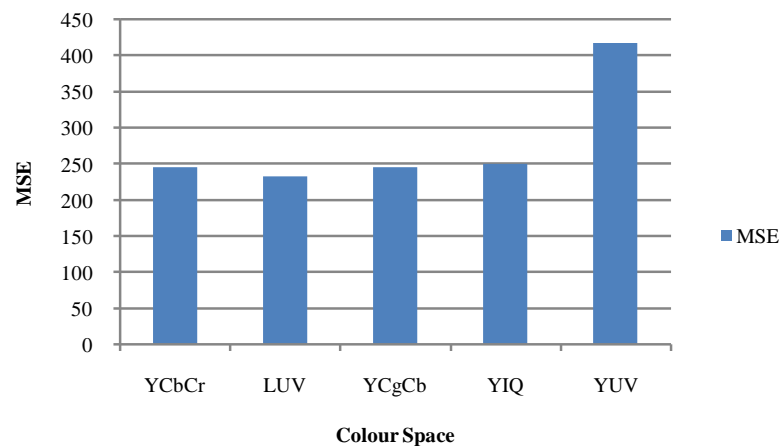

Figure 5 - Colour MSE across all images

It can be noticed that in the case of the reconstructed colour images, there is no clear winner across four colour spaces when the MSE is averaged across all images: YCbCr, Kekre's LUV, Kekre's $\mathrm{YCgCb}$, and YIQ. The YUV colour space clearly underperforms using this technique for both greyscale and colour images and hence is not recommended. The colour space that performs the best is the Kekre's LUV colour space, but not by a large amount. The Kekre's YCgCb colour space, the YIQ colour space, and the $\mathrm{YCbCr}$ colour space give almost identical performance results based on the MSE.

To show the results in more detail, the original greyscale image, the matted greyscale image, and the reconstructed colour image of the first image (baby) is given in figure 6.Some of the images used when implementing this procedure are now reproduced for a more subjective evaluation along with their individual objective evaluations based on the MSE.

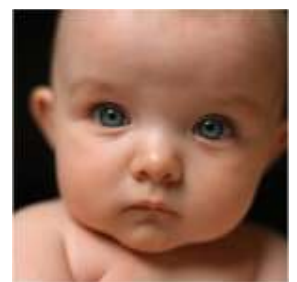

Figure 6a - Original Colour (Baby)
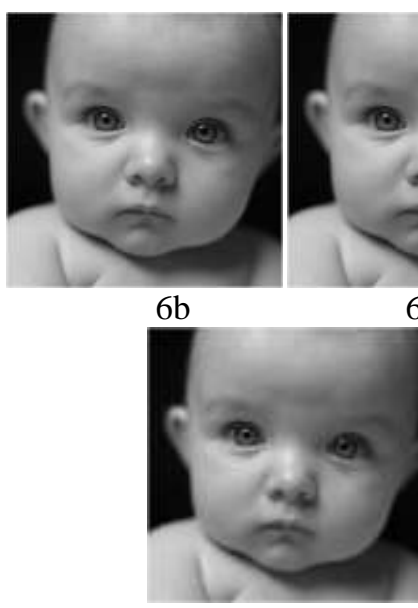

$6 \mathrm{e}$

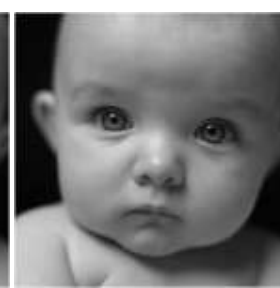

$6 c$

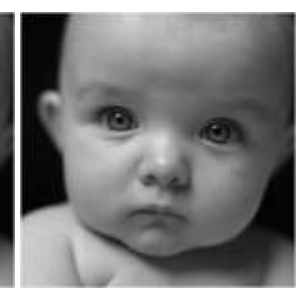

$6 d$

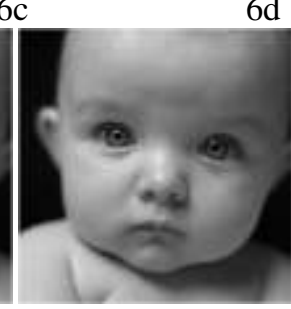

$6 f$

Figure 6b, 6c, 6d, 6e, 6f - Original Greyscales (YCbCr, Kekre's LUV, Kekre's YCgCb, YIQ, YUV)

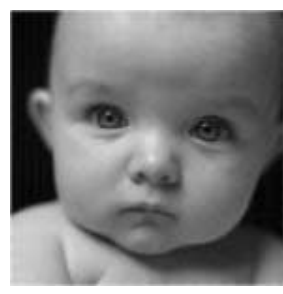

$6 g$

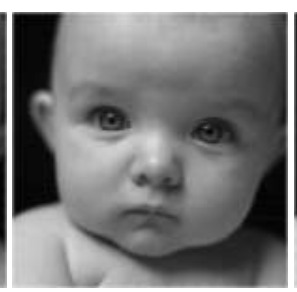

$6 \mathrm{~h}$

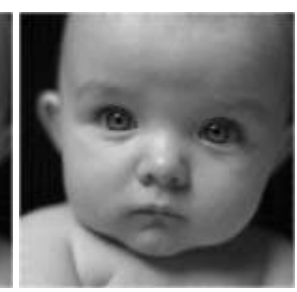

$6 \mathrm{i}$

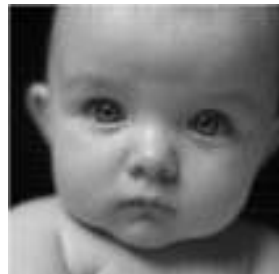

6j

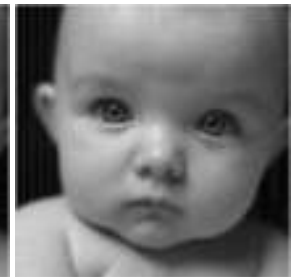

$6 \mathrm{k}$ 
Figure 6g, 6h, 6i, 6j, 6k - Matted Greyscales (YCbCr, Kekre's LUV, Kekre's YCgCb, YIQ, YUV)

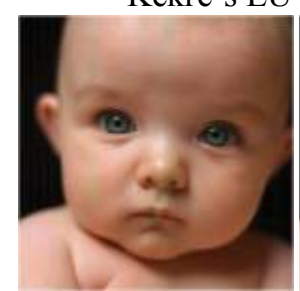

61

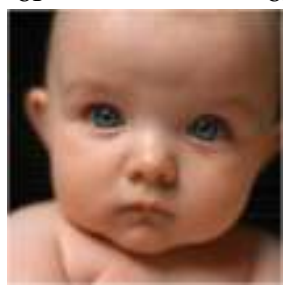

60

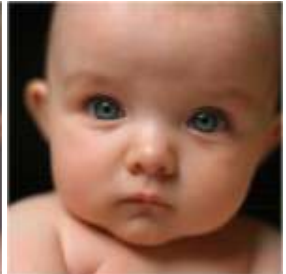

$6 \mathrm{~m}$

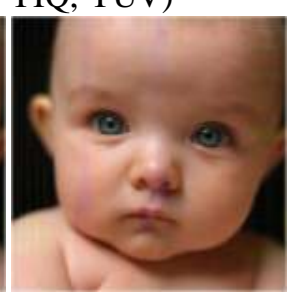

6n

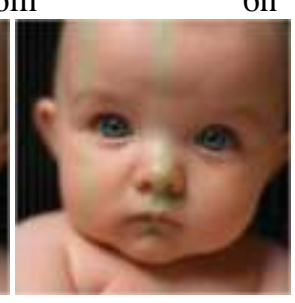

$6 \mathrm{p}$

Figure 61, 6m, 6n, 6o, 6p - Reconstructed Colour (YCbCr, Kekre's LUV, Kekre's YCgCb, YIQ, YUV)

For the image of the baby shown in Figure 6, it is seen that the Kekre's LUV colour space seems to provide the best all round performance, that is, good results are obtained using the Kekre's LUV colour space both at the matted greyscale step, as well as at the reconstructed colour step. For this image, errors in the reconstructed colour image can clearly be perceived as discolouration across the baby's face. In fact, the most evident discolouration is found when the YUV colour space was used. Thus, it appears that the Kekre's LUV colour space outperforms the other colour spaces when the images consist of faces.

For the image of the bus shown in figure 7, on the other hand, the Kekre's YCgCb colour space performs significantly better than the other colour spaces. This is a much more complex image that consists of a non-uniform background and a detailed foreground. Thus, for such images, the Kekre's YCgCb colour space appears to easily win in terms of sheer performance.

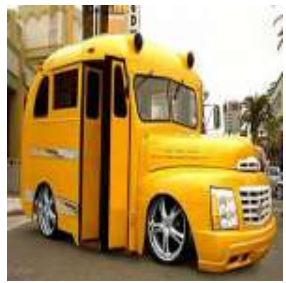

Figure 7a-Original Colour (Bus)

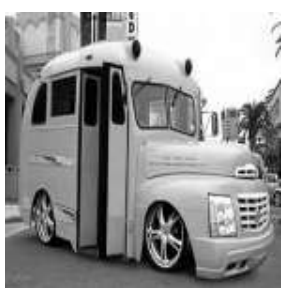

$7 \mathrm{~b}$

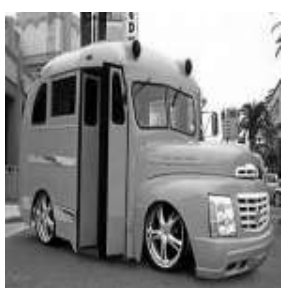

$7 \mathrm{c}$

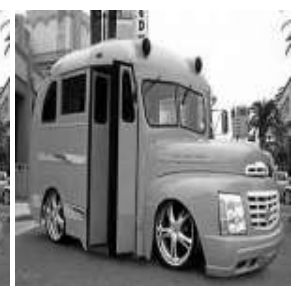

$7 \mathrm{~d}$

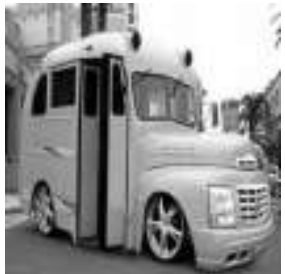

$7 \mathrm{e}$

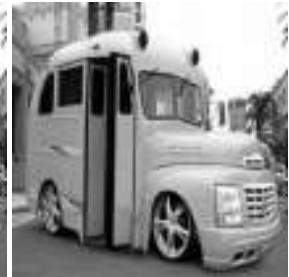

$7 f$
Figure 7b, 7c, 7d, 7e, 7f - Original Greyscales (YCbCr, Kekre's LUV, Kekre's YCgCb, YIQ, YUV)
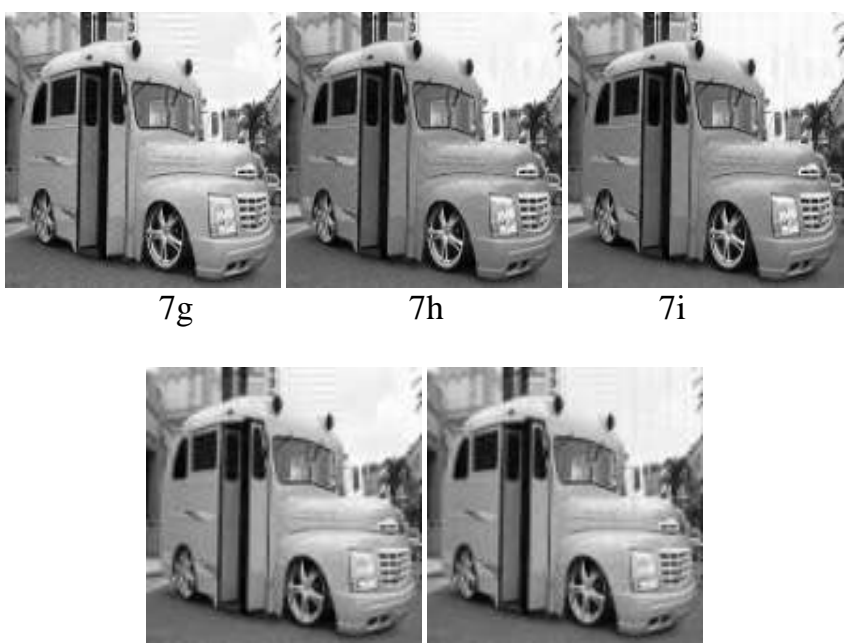

$7 \mathrm{j}$

Figure 7g, 7h, 7i, 7j, 7k - Matted Greyscales (YCbCr, Kekre's LUV, Kekre's YCgCb, YIQ, YUV)
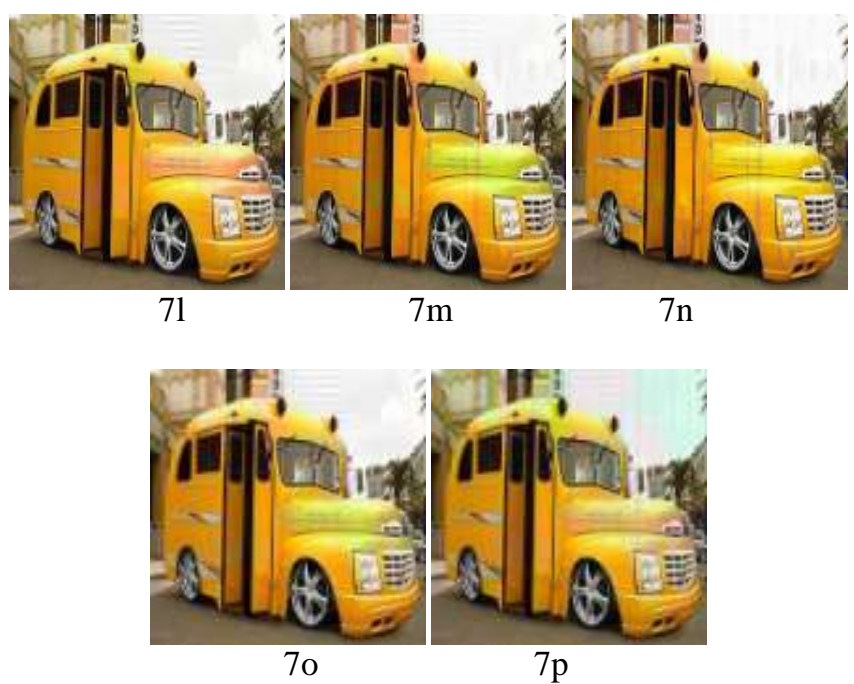

Figure 71, 7m, 7n, 7o, 7p - Reconstructed Colour (YCbCr, Kekre's LUV, Kekre's YCgCb, YIQ, YUV)

The image of the goldfish, shown in figure 8, appears to have no clear winner. Once again, however, the Kekre's LUV colour space has been outperformed by the Kekre's YCgCb colour space and the YCbCr colour space when the reconstructed colour image is subjectively analyzed. However, it must be noted that all five colour spaces have resulted in a certain amount of discolouration of the reconstructed image with the YUV colour space being unacceptable. This image clearly shows that the YUV colour space is unsuited to this technique. No other colour space has caused as much of a discolouration of the white background as the YUV colour space and this can be seen in figure $8 \mathrm{p}$.

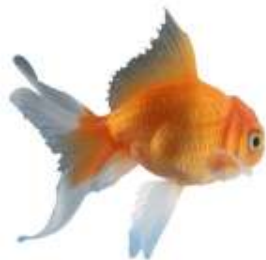

Figure 8a - Original Colour (Goldfish) 
International Journal of Computer Applications (0975 - 8887)

Volume 6-No.7, September 2010

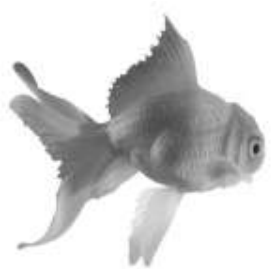

$8 b$

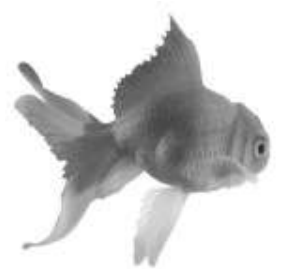

$8 \mathrm{c}$

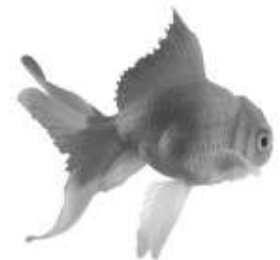

$8 \mathrm{~d}$

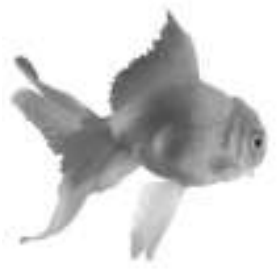

$8 \mathrm{e}$

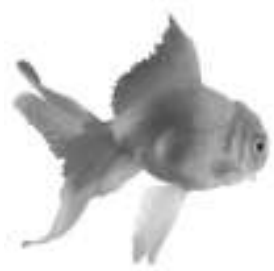

$8 f$
Figure 8b, 8c, 8d, 8e, 8f - Original Greyscales (YCbCr,

Kekre's LUV, Kekre's YCgCb, YIQ, YUV)

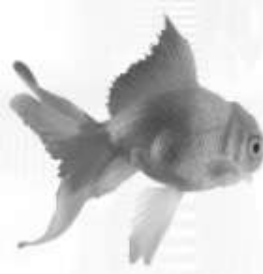

$8 g$

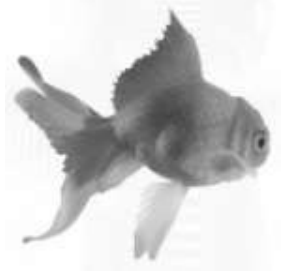

$8 \mathrm{~h}$

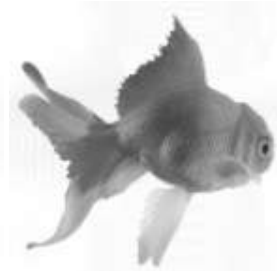

$8 \mathrm{i}$

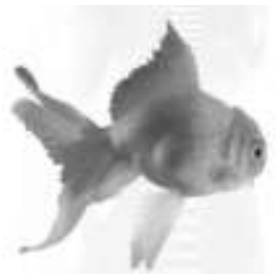

$8 \mathrm{j}$

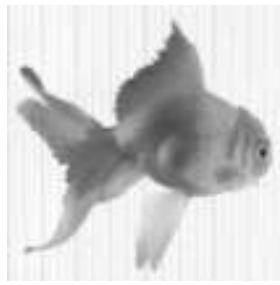

$8 \mathrm{k}$
Figure 8g, 8h, 8i, 8j, 8k - Matted Greyscales (YCbCr, Kekre's LUV, Kekre's YCgCb, YIQ, YUV)

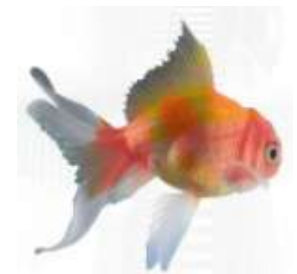

81

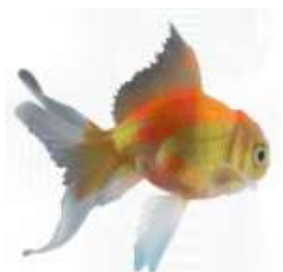

$8 \mathrm{~m}$

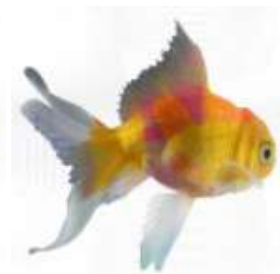

$8 n$

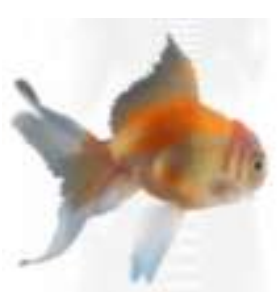

80
$8 \mathrm{p}$

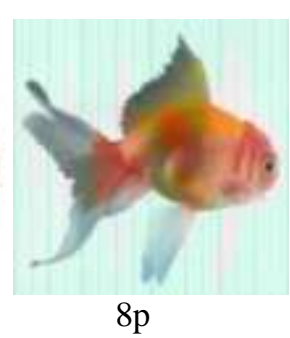

Figure 81, 8m, 8n, 8o, 8p - Reconstructed Colour (YCbCr, Kekre's LUV, Kekre's YCgCb, YIQ, YUV)
Table 3 gives the greyscale MSE values for the three images shown in figures $6-8$. Figure 9 illustrates the same results as a bar chart. Similarly, table 4 gives the colour MSE values for the three images and figure 10 illustrates the same results also as a bar chart.

\begin{tabular}{|c|c|c|c|c|c|}
\hline & YCbCr & $\begin{array}{c}\text { Kekre's } \\
\text { LUV }\end{array}$ & $\begin{array}{c}\text { Kekre's } \\
\text { YCgCb }\end{array}$ & YIQ & YUV \\
\hline Baby & 435.164 & 312.61 & 381.529 & 500.311 & 833.342 \\
\hline Bus & 1108.26 & 965.175 & 840.322 & 1081.61 & 1245.27 \\
\hline Fish & 222.091 & 144.039 & 180.669 & 223.179 & 579.679 \\
\hline
\end{tabular}

Table 3 - MSE between Original Greyscale and Matted

Greyscale versions of 3 images

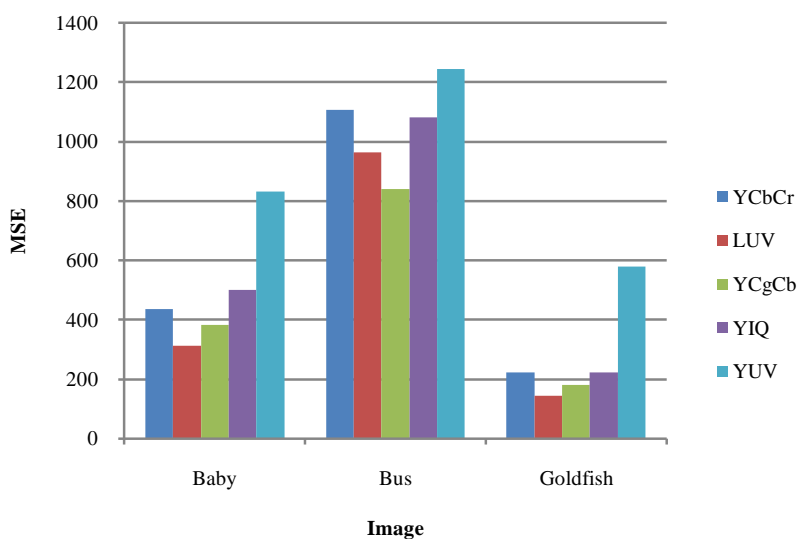

Figure 9 - MSE between Original Greyscale and Matted Greyscale versions of 3 images

\begin{tabular}{|c|c|c|c|c|c|}
\hline & YCbCr & $\begin{array}{c}\text { Kekre's } \\
\text { LUV }\end{array}$ & $\begin{array}{c}\text { Kekre's } \\
\text { YCgCb }\end{array}$ & YIQ & YUV \\
\hline Baby & 202.149 & 197.013 & 204.753 & 199.476 & 234.268 \\
\hline Bus & 647.684 & 567.448 & 562.086 & 591.44 & 745.5 \\
\hline Fish & 162.455 & 161.752 & 154.351 & 202.054 & 602.276 \\
\hline
\end{tabular}

Table 4 - MSE between Original Colour and Recoloured versions of 3 images

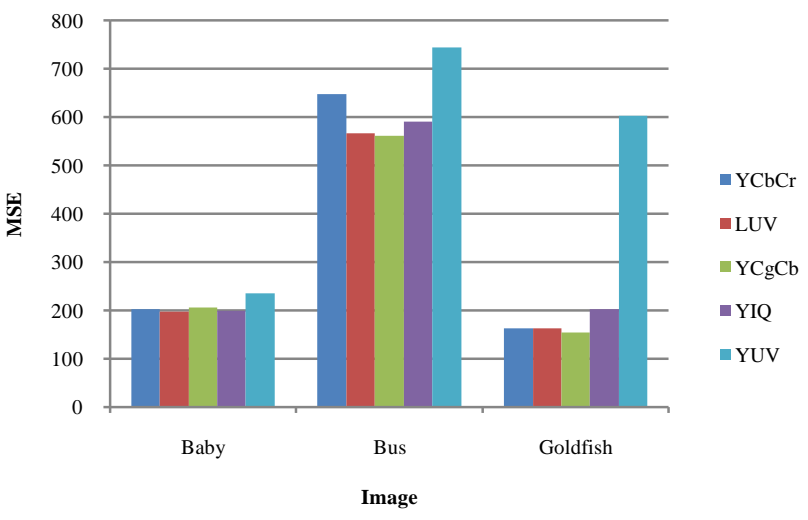

Figure 10 - MSE between Original Colour and Recoloured versions of 3 images

\section{CONCLUSION}

Having analysed this novel technique to embed colour information within a greyscale image using various colour spaces and 
transformations using Haar wavelets for many different categories of images, it can be concluded that while both the Kekre's LUV and the Kekre's $\mathrm{YCgCb}$ colour spaces offer improved performance as compared to the $\mathrm{YCbCr}$ colour space, the difference in performance between the Kekre's LUV colour space and the Kekre's YCgCb colour spaces is less apparent. The results appear to show that the choice between the Kekre's LUV colour space and the Kekre's $\mathrm{YCgCb}$ colour space is image dependent. On the other hand, the YUV colour space can be safely rejected when considering this technique due to its unacceptably poor results across all the various types of images considered. The distinction between the remaining colour spaces is less apparent.

\section{REFERENCES}

[1] E. Reinhard, M. Ashikhmin, B. Gooch, and P. Shirley, "Color transfer between images," IEEE Computer graphics and applications, vol. 21, no. 5, pp. 34-41, September/October 2001.

[2] Dr. H. B. Kekre, Sudeep D. Thepade, Archana Athawale, Adib Parkar, "Using Assorted Color Spaces and Pixel Window Sizes for Colorization of Grayscale Images", ACMInternational Conference and Workshop on Emerging Trends in Technology (ICWET 2010), Thakur College of Engg. And Tech., Mumbai, 26-27 Feb 2010, The paper is uploaded on online ACM Portal.

[3] Ricardo L. de Queiroz, Karen M. Braun, "Color to Gray and Back: Color Embedding into Textured Gray Images", 1464 IEEE TRANSACTIONS ON IMAGE PROCESSING, VOL. 15, NO. 6, JUNE 2006.

[4] Son Lam Fung, A. Bouzerdoum, D. Chai, "A novel skin color model in YCbCr color space and its application to human face detection", In Proc. of International Conference on Image Processing (ICIP-2002), Vol.1, pp. I289-I292.

[5] Hideki Noda, Michiharu Niimi, "Colorization in $\mathrm{YCbCr}$ color space and its application to JPEG images", Pattern Recognition Society Published by Elsevier B.V., Vol.40, number 12, pp.3714-3720, December, 2007.

[6] H. B. Kekre, Sudeep D. Thepade, "Image Blending in Vista Creation using Kekre's LUV Color Space", In SPIT-IEEE Colloquium, SPIT Mumbai, INDIA, Feb 4-5,2008.

[7] H. B. Kekre, Sudeep D. Thepade, "Improving 'Color to Gray and Back' using Kekre's LUV Color Space", IEEE International Advanced Computing Conference 2009 (IACC '09), Thapar University, Patiala, INDIA, 6-7 March 2009.

[8] "Boosting Block Truncation Coding using Kekre's LUV Color Space for Image Retrieval", WASET International Journal of Electrical, Computer and System Engineering (IJECSE), Volume 2, No.3, Summer 2008.

[9] H. B. Kekre, Sudeep D. Thepade, Adib Parkar, "A Comparison of Kekre's Fast Search and Exhaustive Search for various Grid Sizes used for Colouring a Greyscale Image," February 2010.

[10] E. Reinhard, M. Ashikhmin, B. Gooch, and P. Shirley, "Color transfer between images," IEEE Computer graphics and applications, vol. 21, no. 5, pp. 34-41, September/October 2001.

[11] D. L. Ruderman, T. W. Cronin, and C. C. Chiao, "Statistics of cone responses to natural images: implications for visual coding," J. Optical Soc. Of America, vol. 15, no. 8, pp. 2036$2045,1998$.

[12] Dr.H.B.Kekre, Sudeep D. Thepade, Akshay Maloo, "Query by image content using color texture features extracted from Haar wavelet pyramid", International Journal of Computer Applications (IJCA) for the special edition on "Computer
Aided Soft Computing Techniques for Imaging and Biomedical Applications", August 2010. http://www.ijcaonline.org

[13] Dr.H.B.Kekre, Sudeep D. Thepade, Akshay Maloo, "Performance Comparison of Image Retrieval Techniques using Wavelet Pyramids of Walsh, Haar and Kekre Transforms", International Journal of Computer Applications (IJCA) Volume 4, Number 10, August 2010 Edition, pp 1-8, http://www.ijcaonline.org/archives/volume4/number10/866$1216 \mathrm{Mnkn}$

[14] Dr. H. B. Kekre, Sudeep D. Thepade, "Color Traits Transfer to Grayscale Images", IEEE -International Conference on Emerging Trends in Engineering and Technology, ICETET2008, 16-18 July 2008, Raisoni College of Engg., Nagpur. Is uploaded and available online at IEEE Xplore CSDL, ACM Portal.

[15]Dr. H. B. Kekre, Sudeep D. Thepade, Adib Parkar, "Performance Analysis of Kekre's Median Fast Search, Kekre's Centroid Fast Search and Exhaustive Search used for Colouring a Greyscale Image", International Journal of Computer Theory and Engineering (IJCTE), Volume 2, Number 4, August 2010, www.ijcte.org

\section{AUTHOR BIOGRAPHIES}

Dr. H. B. Kekre has received B.E. (Hons.) in Telecomm. Engineering. from Jabalpur University in 1958, M.Tech (Industrial Electronics) from IIT Bombay in 1960, M.S.Engg. (Electrical Engg.) from University of Ottawa in 1965 and Ph.D. (System Identification) from IIT Bombay in $1970 \mathrm{He}$ has worked as Faculty of Electrical Engg. and then HOD Computer Science and Engg. at IIT Bombay. For 13 years he was working as a professor and head in the Department of Computer Engg. at Thadomal Shahani Engineering. College, Mumbai. Now he is Senior Professor at MPSTME, SVKM's NMIMS University. He has guided $17 \mathrm{Ph} . \mathrm{Ds}$, more than 100 M.E./M.Tech and several B.E./B.Tech projects. His areas of interest are Digital Signal processing, Image Processing and Computer Networking. He has more than 320 papers in National / International Conferences and Journals to his credit. He was Senior Member of IEEE. Presently $\mathrm{He}$ is Fellow of IETE and Life Member of ISTE Recently ten students working under his guidance have received best paper awards and two have been conferred Ph.D. degree of SVKM's NMIMS University. Currently 10 research scholars are pursuing $\mathrm{Ph} . \mathrm{D}$. program under his guidance.

Sudeep D. Thepade has Received B.E.(Computer) degree from North Maharashtra University with Distinction in 2003. M.E. in Computer Engineering from University of Mumbai in 2008 with Distinction, currently pursuing Ph.D. from SVKM's NMIMS, Mumbai. He has about than 07 years of experience in teaching and industry. He was Lecturer in Dept. of Information Technology at Thadomal Shahani Engineering College, Bandra(w), Mumbai for nearly 04 years. Currently working as Associate Professor in Computer Engineering at Mukesh Patel School of Technology Management and Engineering, SVKM's NMIMS University, Vile Parle(w), Mumbai, INDIA. He is member of International Association of Engineers (IAENG) and International Association of Computer Science and Information Technology (IACSIT), Singapore. His areas of interest are Image Processing and Computer Networks. He has about 75 papers in National/International Conferences/Journals to his credit with a Best Paper Award at International Conference SSPCCIN-2008, Second Best Paper Award at ThinkQuest-2009 National Level paper presentation competition for faculty and Best Paper Award at Springer International Conference ICCCT-2010.

Adib Parkar is pursuing a Bachelors (B.E.) degree in Computer Science from Thadomal Shahani Engineering College in Mumbai, India. He has been an active IEEE Student Member for 3 years and is also a member of the Computer Society of India. His areas of interest lie in the fields of Image Processing and Artificial Intelligence. 